\title{
Quantum storage of optical signals and coherent manipulation of quantum states based on electromagnetically induced transparency
}

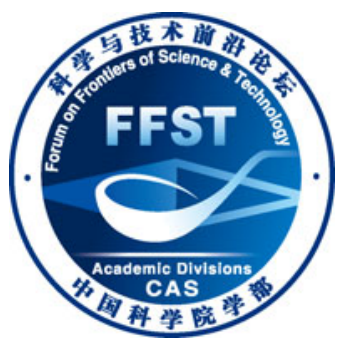

\author{
WANG Hai ${ }^{*}$, XIE ChangDe \& PENG KunChi \\ State Key Laboratory of Quantum Optics and Quantum Optics Devices, Institute of Opto-Electronics, Shanxi University, Taiyuan 030006, China
}

Received December 12, 2011; accepted February 9, 2012

\begin{abstract}
Electromagnetically induced transparency (EIT) techniques are important tools for the storage of the quantum states of light fields in atomic ensembles and for enhancement of the interaction between photons. In this paper, we briefly summarize the recent experimental studies conducted by our group on enhanced cross-phase modulation based on double EIT effects, the quantum interference of stored dual-channel spin-wave excitations and the coherent manipulation of the spin wave vector for the polarization of photons in a single tripod atomic system. The work presented here has potential application in the developing field of quantum information processing.
\end{abstract}

electromagnetically induced transparency (EIT), atomic ensembles, quantum storage, enhanced cross-phase modulation, coherent manipulation of the spin wave

Citation: Wang H, Xie C D, Peng K C. Quantum storage of optical signals and coherent manipulation of quantum states based on electromagnetically induced transparency. Chin Sci Bull, 2012, 57: 1893-1902, doi: 10.1007/s11434-012-5090-6

Photons travel fast and are insensitive to the surrounding environment, thus they are often used as carriers of information. However, photons are difficult to trap and store, and the interactions between them are quite weak, which prevent their use in some applications in quantum information processing (QIP). In recent years, electromagnetically induced transparency (EIT) and its related effects have been studied in depth both theoretically and experimentally [1,2]. These studies have shown that EIT effects in three-level or multi-level atomic systems can be applied to significantly reduce the group velocity of light, to trap and store photons using an atomic ensemble and to greatly enhance the optical cross-Kerr nonlinearity.

The typical EIT effects can be demonstrated using a three-level $\Lambda$-type atomic system, as shown in Figure 1 , in which a strong coupling laser beam couples to the atomic transition from the $|s\rangle$ state to the $|e\rangle$ state and a weak probe beam couples to the atomic transition from the $|g\rangle$

*Corresponding author (email: wanghai@sxu.edu.cn) state to the $|e\rangle$ state. Under these conditions, the coherent superposition states $|s\rangle$ and $|g\rangle$ are produced and a transparency window for the probe beam appears at a two-photon resonance (Figure 2). This effect is called electromagnetically induced transparency (EIT) or dark resonance [2].

Based on the EIT dynamic process, the quantum states of light have been stored successfully in atomic ensembles [3,4]. Recent studies show that EIT and its related effects are important tools in QIP [5-9]. Also, EIT effects can be

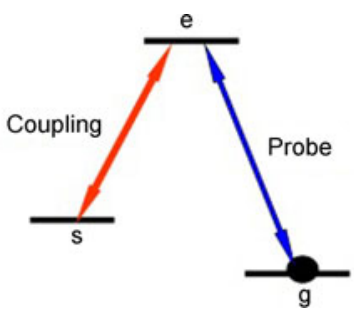

Figure 1 (Color online) Three-level $\Lambda$-type atomic system. 


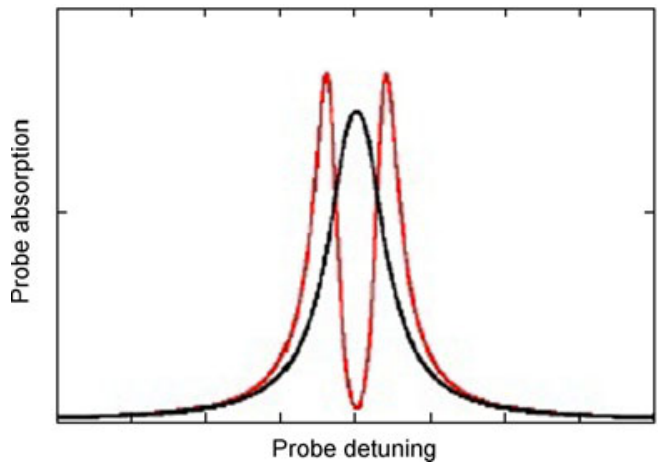

Figure 2 (Color online) Transmission spectra corresponding to the cases without EIT (black) and with EIT (red).

used to enhance the nonlinear optical interaction between photons [1]. Because of the reduced linear absorption at resonance, EIT effects can be used in resonant nonlinear optical processes, in which the nonlinear optical coefficients of the atomic media are much larger than those under the normal off-resonant conditions [1].

In this paper, we briefly review the experimental studies of enhanced cross-phase modulation based on double EIT [10], the quantum interference of stored dual-channel spinwave excitations [11], and the coherent manipulation of the spin wave vector for the polarization of photons [12] in a four-level tripod atomic system, which were conducted by our group over the last few years.

\section{Enhanced cross-phase modulation based on double EIT in a four-level tripod atomic system}

Cross-Kerr nonlinearity, also known as cross-phase modulation (XPM), has been paid increasing attention because potential applications have been found in demonstrating quantum phase gates [13-15], generating quantum entanglement of single photons [16], constructing a neardeterministic controlled-not gate [17], performing nondestructive Bell-state detection [18], and implementing quantum computation in a new protocol [19]. However, the absence of sufficient XPM in conventional media is an obstacle to its application in QIP. EIT technology provides a promising avenue to solve this problem [1]. The large cross-Kerr nonlinearity in a four-level N-type EIT system has been theoretically predicted [20] and experimentally demonstrated in cold atoms [21]. However, the large cross-Kerr nonlinearity between the probe and trigger (signal) pulses may not always occur in the N-type EIT system because of their group velocity mismatch [22-24]. Further experimental research on this effect is highly anticipated. With this motivation, we carried out an experimental study of double EIT and the enhanced cross-phase modulation between the two fields in a four-level tripod EIT system of the D1 line of ${ }^{87} \mathrm{Rb}$ atoms [10].
The four-level tripod atomic system is shown in Figure 3. The probe field $E_{P}$ of frequency $\omega_{P}$ is left-circularlypolarized $\left(\sigma^{-}\right)$, and coupling occurs at the transitions from levels $\left|a_{i+1}\right\rangle$ to $\left|e_{i}\right\rangle(i=1,2)$. The coupling field $E_{C}$ of frequency $\omega_{C}$ is also $\sigma^{-}$-polarized, to drive the transitions from levels $\left|b_{i+2}\right\rangle$ to $\left|e_{i}\right\rangle(i=1,2,3)$. The trigger field $E_{T}$ of frequency $\omega_{T}$ is right-circularly-polarized $\left(\sigma^{+}\right)$and couples to the transitions of levels $\mid b_{i}>$ to $\left|e_{i}\right\rangle(i=1,2,3)$. In this case, the system is coherently prepared into two four-level tripod-type systems [25]. One system is formed by the levels $\left|a_{2}>-\right| b_{3}>-\left|b_{1}>-\right| e_{1}>(\mathrm{S} 1)$, and the other system is formed by the levels $\left|a_{3}>-\right| b_{4}>-\left|b_{2}>-\right| e_{2}>(\mathrm{S} 2)$. The $\sigma^{-}$probe transition and $\sigma^{+}$trigger transition share a common excited state, $\mid e_{1}>\left(\mid e_{2}>\right)$, which induces coupling between the probe and trigger fields in such a coherently prepared tripod system, and the cross-Kerr phase modulation between the two fields is then enhanced.

The double EIT windows for the probe and trigger fields are shown in Figure 4. These windows are obtained by scanning the probe frequency across $\omega_{a e}$ (Figure 4(a)) and scanning the trigger frequency around $\omega_{b e}$ (Figure 4(b)).

The measured XPM phase shifts and the transmissions at the different coupling detuning $\left(\Delta_{C}\right)$ values under the conditions of weak probe and trigger fields $\left(P_{p} \approx P_{T} \approx 14 \mu \mathrm{W}\right)$ are shown in Figure 5. The curves A and B in Figure 5 plot the measured XPM coefficients $n_{P}^{(2)}$ and $n_{T}^{(2)}$ obtained at $\Delta_{P}-\Delta_{C} \approx-0.5 \mathrm{MHz}$ as a function of $\Delta_{C}$. The curves $\mathrm{C}$ and $\mathrm{D}$ in the inset of Figure 5 plot the simultaneously measured transmission values, also at $\Delta_{P}-\Delta_{C} \approx-0.5 \mathrm{MHz}$. For increasing $\Delta_{C}$, the absolute values of $n_{P}^{(2)}$ and $n_{T}^{(2)}$ at $\Delta_{P}-\Delta_{C} \approx-0.5 \mathrm{MHz}$ decrease, and the accompanying transmission values of the probe and trigger beams increase, which allows the choice of the appropriate coupling frequency detuning to achieve a substantial XPM phase shift with smaller absorption. Figure 5 shows that $n_{T}^{(2)}$ is much larger than $n_{P}^{(2)}$. This asymmetry of $n_{T}^{(2)}$ and $n_{P}^{(2)}$ does

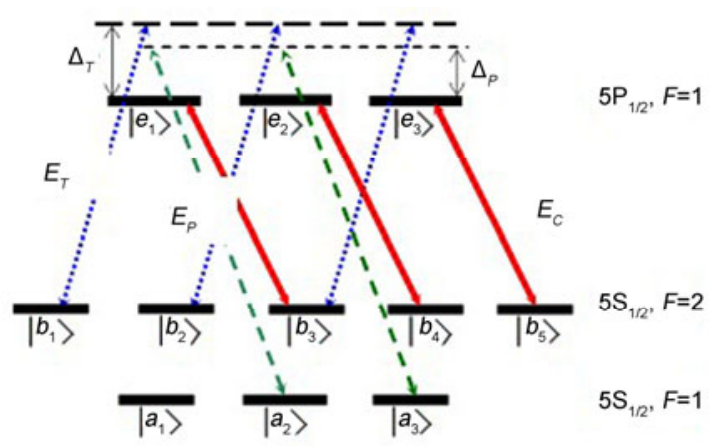

Figure 3 (Color online) Relevant atomic energy level diagram of the D1 line in the ${ }^{87} \mathrm{Rb}$ atom 

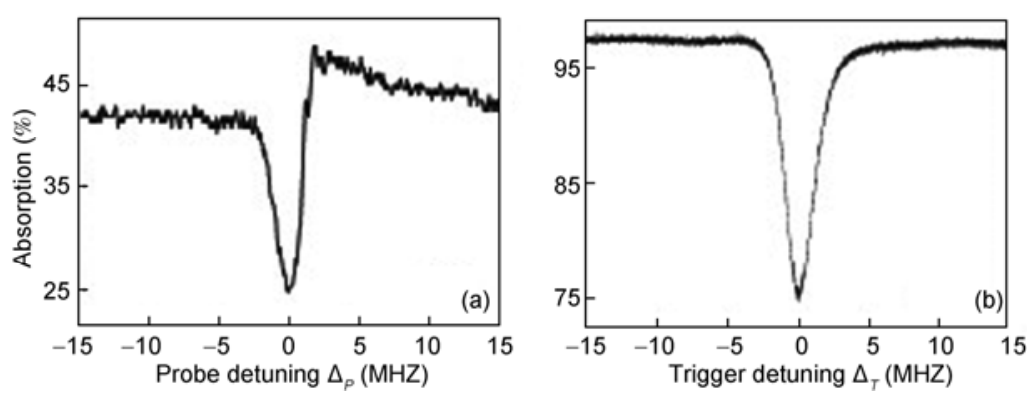

Figure 4 The measured simultaneous EIT windows for probe (a) and trigger (b) beams under the action of a coupling beam.

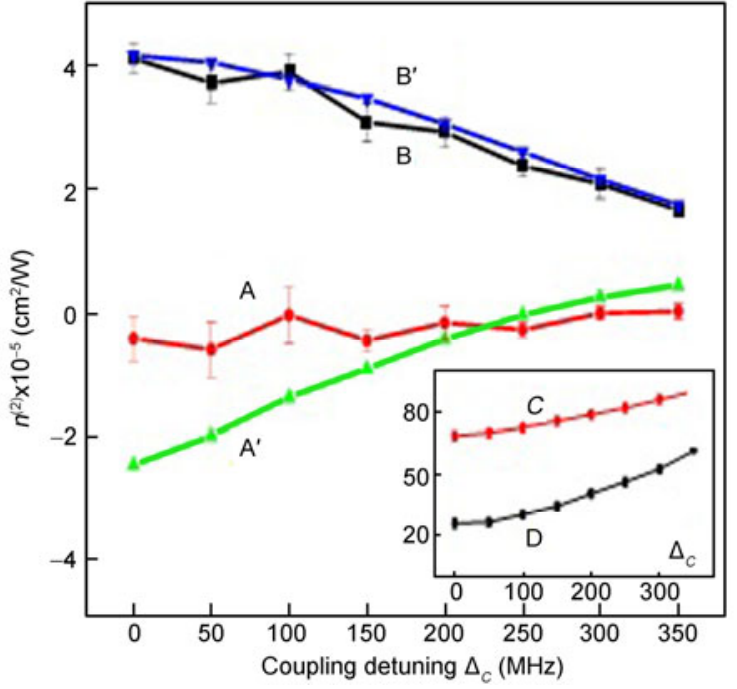

Figure 5 (Color online) The probe $\left(\mathrm{A}, \mathrm{A}^{\prime}\right)$ and trigger $\left(\mathrm{B}, \mathrm{B}^{\prime}\right)$ XPM coefficients as functions of coupling detuning.

not agree with the predictions in $[14,15]$, in which $n_{T}^{(2)}$ and $n_{P}^{(2)}$ are symmetrical and can be exchanged each other. One possible reason for this disagreement is that the large difference between the probe Rabi frequencies $\left(\Omega_{P 1}=\Omega_{P 2}=\right.$ $1.15 \mathrm{MHz})$ and the trigger Rabi frequencies $\left(\Omega_{T 1}=\right.$ $2.83 \mathrm{MHz}, \Omega_{T 2}=2 \mathrm{MHz}$ ) results in a difference between the populations of the two ground states $\mid a_{2}>$ and $\left|b_{1}\right\rangle\left(\left|a_{3}\right\rangle\right.$ and $\left.\mid b_{2}>\right)$. The populations calculated using eq. (3) in [25] are $\rho_{a 2, a 2} \approx 0.38, \quad \rho_{a 3, a 3} \approx 0.3, \quad \rho_{b 1, b 1} \approx 0.12$ and $\rho_{b 2, b 2} \approx$ 0.2 for $\Omega_{P 1}=\Omega_{P 2}=1.15 \mathrm{MHz}, \Omega_{T 1}=2.83 \mathrm{MHZ}$ and $\Omega_{T 2}=2 \mathrm{MHz}$. The curves $\mathrm{A}^{\prime}$ and $\mathrm{B}^{\prime}$ are the calculated fitting curves for $n_{P}^{(2)}$ and $n_{T}^{(2)}$ at $\Delta_{P}-\Delta_{C}=-0.5 \mathrm{MHz}$ using eq. (1) in [10], and are in reasonable agreement with the experimental results.

The enhanced XPM in the tripod system presented here is based on simultaneous EIT of the probe and trigger fields, and thus both the probe and trigger absorptions are small. For example, the trigger XPM coefficient can reach $2 \times 10^{-5}$ $\mathrm{cm}^{2} / \mathrm{W}$ with a transmission of $\sim 60 \%$ (see Figure 4 ). Although the conditional phase shift, which is defined as
$\Phi_{P}^{N}+\Phi_{T}^{N} \quad$ [13] in a quantum phase gate (QPG), still cannot reach $\sim \pi$ under our experimental conditions, if the laser linewidths of the probe and trigger beams are suppressed down to $5 \mathrm{kHz}$, a conditional phase shift $\left(\Phi_{P}^{N}+\Phi_{T}^{N}\right)$ based on the XPM between the two single-photon pulses will reach $1.2 \mathrm{rad}$ for $\Omega_{C}=30 \mathrm{MHz}$, which may allow us to perform the QPG operation.

\section{Quantum interference of stored dual-channel spin-wave excitations in a single tripod system}

Using the EIT dynamic process, photons can be transferred into a single-channel collective spin-wave excitation (SWE) or atomic coherence (Zeeman coherence) in a typical EIT three-level $\Lambda$-type atomic system [2,9]. In storage experiments for classical and quantum optical signals, a prepared three-level $\Lambda$-type atomic system is used as the storage medium and a single-channel spin coherence is involved. The retrieved signal thus exhibits monotonic decay with a storage time on a millisecond time scale in a cold atomic cloud [9], and on a time scale of seconds in an atomic lattice [26] or Bose-Einstein condensate (BEC) [27]. In most atomic memory experiments, the atomic system includes multiple $\Lambda$-type subsystems and thus the dark-state polaritons include many different Zeeman coherences. Because different Zeeman coherences have different Larmor precession periods, the dark-state polaritons experience a "collapse and revival" phenomenon [28] because of the interference between the multiple SWEs. Schemes for dual-channel storage, i.e. storing an optical signal simultaneously into two adjacent SWEs in the four-level inverted-Y [29] or tripod [30] atomic configurations, have been proposed, in which the manipulation of the storage and release of the optical signals can be described well by the generalized dark-state polariton (GDSP) dynamics [29].

Recently, we experimentally demonstrated a dualchannel memory where the retrieval efficiency varied with the relative phase between the two reading light beams in a single four-level tripod system [11]. The scheme of the dualchannel memory in a single four-level tripod system is 


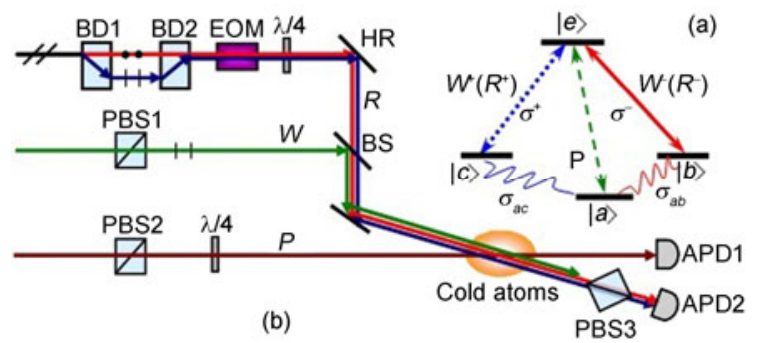

Figure 6 (Color online) Experimental setup.

shown in Figure 6. The weak signal beam (P) is simultaneously stored in the dual-channel coherent SWEs ( $\tilde{\sigma}_{c a}$ and $\tilde{\sigma}_{b a}$ ) by switching off the two circularly-polarized writing beams and can then be read at a later time by switching on the two circularly-polarized beams $\left(R^{+}, R^{-}\right)$with a certain relative phase $\delta_{R}=\phi_{R}^{+}-\phi_{R}^{-}$. Because quantum interference is induced by the light-matter interaction between the two stored collective SWEs, the total readout signal shows a maximum or minimum value depending on the relative phase $\delta_{R}$ between the two reading light beams. By varying $\delta_{R}$, the total readout signal shows a sinusoidal interference pattern.

A simple theoretical description of the optical storage/ retrieval processes in the tripod system is given as follows. The generalized dark-state polaritons, including a quantum field $\hat{\varepsilon}_{p}(z, t)$ and a total collective atomic spin wave $\hat{S}(z, t)$, can be written as

$$
\hat{\Psi}(z, t)=\cos \theta(t) \cdot \hat{\varepsilon}_{p}(z, t)-\sin \theta(t) \cdot \sqrt{N} \hat{S}(z, t),
$$

where

$$
\begin{aligned}
\hat{S}(z, t)= & \frac{\left|\Omega_{C}^{+}(t)\right|}{\sqrt{\left|\Omega_{C}^{+}(t)\right|^{2}+\left|\Omega_{C}^{-}(t)\right|^{2}}} \mathrm{e}^{-i \phi_{C}^{+}} \tilde{\sigma}_{c a}(z, t) \\
& +\frac{\left|\Omega_{C}^{-}(t)\right|}{\sqrt{\left|\Omega_{C}^{+}(t)\right|^{2}+\left|\Omega_{C}^{-}(t)\right|^{2}}} \mathrm{e}^{-i \phi_{C}^{-}} \tilde{\sigma}_{b a}(z, t) .
\end{aligned}
$$

The mixing angle is given by $\operatorname{tg} \theta(t)=$ $\frac{g \sqrt{N}}{\sqrt{\left|\Omega_{C}^{+}(t)\right|^{2}+\left|\Omega_{C}^{-}(t)\right|^{2}}} ; \quad\left|\Omega_{c}^{+}(t)\right|$ and $\left|\Omega_{c}^{-}(t)\right|$ (where $C$ denotes $W$ or $R$ ) are the amplitudes of the Rabi frequencies for the right- and left-circularly-polarized writing $\left(W^{+}\right.$and $\left.W^{-}\right)$or reading $\left(R^{+}\right.$and $\left.R^{-}\right)$beams, respectively; $\phi_{C}^{+}$and $\phi_{C}^{-}$are the phases of the writing $\left(W^{+}\right.$and $\left.W\right)$ or reading $\left(R^{+}\right.$and $\left.R^{-}\right)$fields, respectively. $\tilde{\sigma}_{c a}\left(\tilde{\sigma}_{b a}\right)$ is the spin coherence between the states $\langle c|$ and $\langle a|(\langle b|$ and $\langle a|)$. In the storage process, the writing beam (including the two components $\Omega_{w}^{+}(t)$ and $\left.\Omega_{w}^{-}(t)\right)$ is adiabatically switched off over the time interval $\left[t_{0}, t_{1}\right]$ as the probe pulse enters into the cold atomic ensemble, and so the probe field $\hat{\varepsilon}_{P}^{i n}(z, t)$ is mapped onto the total $\operatorname{SWE} \hat{S}_{W}(z, t)$ and stored in the cold atomic ensemble. Under the condition where $\left|\Omega_{W}^{+}(t)\right|=\left|\Omega_{W}^{-}(t)\right|$, this total $\operatorname{SWE} \hat{S}_{W}(z, t)$ can be written from eq. (2), as

$$
\begin{aligned}
\hat{S}_{W}\left(z, t_{1}\right)= & \frac{1}{\sqrt{2}} \tilde{\sigma}_{c a}\left(z, t_{1}\right) \mathrm{e}^{-i \phi_{w}^{+}} \\
& +\frac{1}{\sqrt{2}} \tilde{\sigma}_{b a}\left(z, t_{1}\right) \mathrm{e}^{-i \phi_{w}^{-}},
\end{aligned}
$$

where $\tilde{\sigma}_{c a}\left(z, t_{1}\right)$ and $\tilde{\sigma}_{b a}\left(z, t_{1}\right)$ are two collective SWEs and are defined as $\tilde{\sigma}_{\beta \alpha}(z, t)=\frac{1}{N_{z}} \sum_{j=1}^{N_{j}} \tilde{\sigma}_{\beta \alpha}^{j}(t)=F(z, t) \hat{\sigma}_{\beta \alpha} \mathrm{e}^{i \omega_{\alpha \beta}(z-c t)}$ $\times \hat{\sigma}_{\beta \alpha}=|\beta\rangle\langle\alpha|$ is a spin flip operator $(\alpha=a, \beta=b$ or $c)$ and $F(z, t)$ is the normalized distribution function for the SWE, which is related to $\hat{\varepsilon}_{P}^{\text {in }}(z, t)$. According to the dark-state polariton theory [2] in the case of $\theta \approx \pi / 2$ (see eq. (1)), the signal field $\hat{\varepsilon}_{P}^{i n}(z, t)$ is mapped into the collective SWE $\hat{S}_{w}(z, t)$, and the two calculated SWEs are

$$
\tilde{\sigma}_{b a}\left(z, t_{1}\right) \propto \frac{1}{\sqrt{2}} \varepsilon_{P}^{i n}\left(z-z_{01}, t_{0}\right) \hat{\sigma}_{b a} \mathrm{e}^{i \phi_{\bar{W}}^{\bar{T}}},
$$

and

$$
\tilde{\sigma}_{c a}\left(z, t_{1}\right) \propto \frac{1}{\sqrt{2}} \varepsilon_{P}^{i n}\left(z-z_{01}, t_{0}\right) \hat{\sigma}_{c a} \mathrm{e}^{i \phi_{W}^{\dagger}},
$$

where $\varepsilon_{P}^{\text {in }}\left(z-z_{01}, t_{0}\right)$ is the slowly-varying amplitude of the input probe field at $z-z_{01} \quad\left(\right.$ where $\left.z_{01}=\int_{t_{0}}^{t_{1}} \mathrm{~d} t^{\prime} v_{g}\left(t^{\prime}\right)\right)$ and time $t=t_{0}$. In a finite magnetic field, the atomic coherences (SWEs) $\tilde{\sigma}_{b a}\left(z, t_{1}\right)$ and $\tilde{\sigma}_{c a}\left(z, t_{1}\right)$ experience a Larmor precession, and after a storage time interval $\tau=t_{2}-t_{1}$, they become

$$
\tilde{\sigma}_{b a}\left(z, t_{2}\right) \propto \frac{1}{\sqrt{2}} \varepsilon_{P}^{i n}\left(z-z_{01}, 0\right) \hat{\sigma}_{b a} \mathrm{e}^{i \phi_{W}^{-}+i 2 \Omega_{L} \tau}
$$

and

$$
\tilde{\sigma}_{c a}\left(z, t_{2}\right) \propto \frac{1}{\sqrt{2}} \varepsilon_{P}^{i n}\left(z-z_{01}, 0\right) \hat{\sigma}_{c a} \mathrm{e}^{i \phi_{W}^{+}} .
$$

$\Omega_{L}=g_{F} \mu_{B} B / \hbar$ is the Larmor procession frequency, and $2 \Omega_{L}$ is the frequency shift between the Zeeman sublevels $|c\rangle \quad(|F=2, m=-1\rangle)$ and $|b\rangle \quad(\mid F=2$, $m=+1\rangle$ ) in the magnetic field $B$. At time $t_{2}$, if the two reading beams, $\left|\Omega_{R}^{+}(t)\right| \mathrm{e}^{-i \phi_{R}^{+}}$and $\left|\Omega_{R}^{-}(t)\right| \mathrm{e}^{-i \phi_{R}^{-}} \quad$ (with $\left.\left|\Omega_{R}^{+}(t)\right|=\left|\Omega_{R}^{-}(t)\right|\right)$, are used to read the two-channel SWEs stored in the tripod atomic medium, then the collective SWE $\hat{S}(z, t)$ becomes 


$$
\begin{aligned}
& \tilde{S}_{R}\left(z, t_{2}\right)=\frac{1}{\sqrt{2}} \tilde{\sigma}_{c a}\left(z, t_{2}\right) \mathrm{e}^{-i \phi_{R}^{+}}+\frac{1}{\sqrt{2}} \tilde{\sigma}_{b a}\left(z, t_{2}\right) \mathrm{e}^{-i \phi_{R}^{-}} \\
& =\frac{1}{2} \varepsilon_{p}^{i n}\left(z-z_{01}, t_{0}\right)\left(\hat{\sigma}_{c a} \mathrm{e}^{i \phi_{w}^{+}-i \phi_{R}^{+}}+\hat{\sigma}_{b a} \mathrm{e}^{i \phi_{w}^{-}-i \phi_{R}^{-}+i 2 \Omega_{L} \tau}\right),
\end{aligned}
$$

which will be turned into the released total optical signal. The total readout signal can then be written as

$$
\begin{aligned}
& \varepsilon_{P}^{\text {out }}(z, t) \propto\left\langle\hat{S}_{R}\left(z-z_{02}, t_{2}\right)\right\rangle \\
& \propto \frac{1}{2} \varepsilon_{P}^{i n}\left(z-z_{0}, t_{0}\right)\left(\mathrm{e}^{-i \delta_{R}+i \delta_{W}-i 2 \Omega_{L} \tau}+1\right),
\end{aligned}
$$

where $z_{02}=\int_{t_{2}}^{t} v_{g}\left(t^{\prime}\right) \mathrm{d} t^{\prime}, \quad z_{0}=z_{01}+z_{02}, \quad \delta_{R}=\phi_{R}^{+}-\phi_{R}^{-}$ and $\delta_{w}=\phi_{w}^{+}-\phi_{w}^{-}$.

The individual signals $\varepsilon_{P^{+}}^{\text {out }}$ and $\varepsilon_{P^{-}}^{\text {out }}$ retrieved separately from the two memory channels $\left(\tilde{\sigma}_{c a}\right.$ and $\left.\tilde{\sigma}_{b a}\right)$ can be easily calculated using eqs. (6) and (7) under conditions of $\Omega_{R}^{-}(t)=0 \quad$ or $\quad \Omega_{R}^{+}(t)=0 \quad$ with $\quad\left|\varepsilon_{P^{+}}^{\text {out }}(z, t)\right|=\left|\varepsilon_{P^{-}}^{\text {out }}(z, t)\right|=$ $\varepsilon_{\text {single }}^{\text {out }}(z, t) \propto \frac{1}{\sqrt{2}} \varepsilon_{P}^{\text {in }}\left(z-z_{0}, t_{0}\right)$. The total retrieved signal intensity can be expressed as

$$
\left|\varepsilon_{P}^{\text {out }}\right|^{2} \propto 2\left|\varepsilon_{\text {single }}^{\text {out }}\right|^{2} \cos ^{2}\left(\frac{\delta_{R}}{2}-\frac{\delta_{W}}{2}+\Omega_{L} \tau\right),
$$

where $\varepsilon_{\text {single }}^{\text {out }}$ denotes the amplitude of the readout signal retrieved from a single channel (only with $\Omega_{R}^{+}(t)$ or
$\left.\Omega_{R}^{-}(t)\right)$. From eq. (8), we can easily see that when $\Delta=\delta_{R}-\delta_{\mathrm{W}}+2 \Omega_{L} \tau=0 \quad$ or $\quad \pi, \quad\left|\varepsilon_{P}^{\text {out }}\right|^{2} \approx 2\left|\varepsilon_{\text {single }}^{\text {out }}\right|^{2} \quad$ or $\left|\varepsilon_{P}^{\text {out }}\right|^{2}=0$, i.e. the total optical readout signal shows the constructive or destructive interference.

The experiment was carried out using a standard magneto-optical trap with ${ }^{87} \mathrm{Rb}$ atoms. The atoms are prepared in an ideal tripod system by selectively pumping the atomic population into the $5 \mathrm{~S}^{1 / 2}, F=1, m=+1$ state [31]. The experimentally prepared tripod system can be described well using the simple energy diagram shown in Figure 6(a), and the configurations of the writing, reading, and signal beams are shown in Figure 6(b), with all of the beams split from a single high-power diode laser. The relative phase difference is introduced by separating the horizontal and vertical polarization components of the reading beam, and is controlled using the electro-optic modulator (EOM).

Figure 7(a) shows the stored and retrieved signals from a single (left) channel (blocking the right writing/reading beams), which corresponds to the typical $\Lambda$-type system. Following the standard procedure for optical storage and retrieval, the retrieved signal is read after a $380 \mathrm{~ns}$ time delay (right peak). The right channel shows the same behavior when used singly. When both the left and right writing beams are turned on and have the same power $\left(\left|\Omega_{w}^{+}(t)\right|=\right.$ $\left.\left|\Omega_{w}^{-}(t)\right|\right)$, as in the single-channel case, the optical signal is stored simultaneously in two coherent SWEs ( $\tilde{\sigma}_{c a}$ and

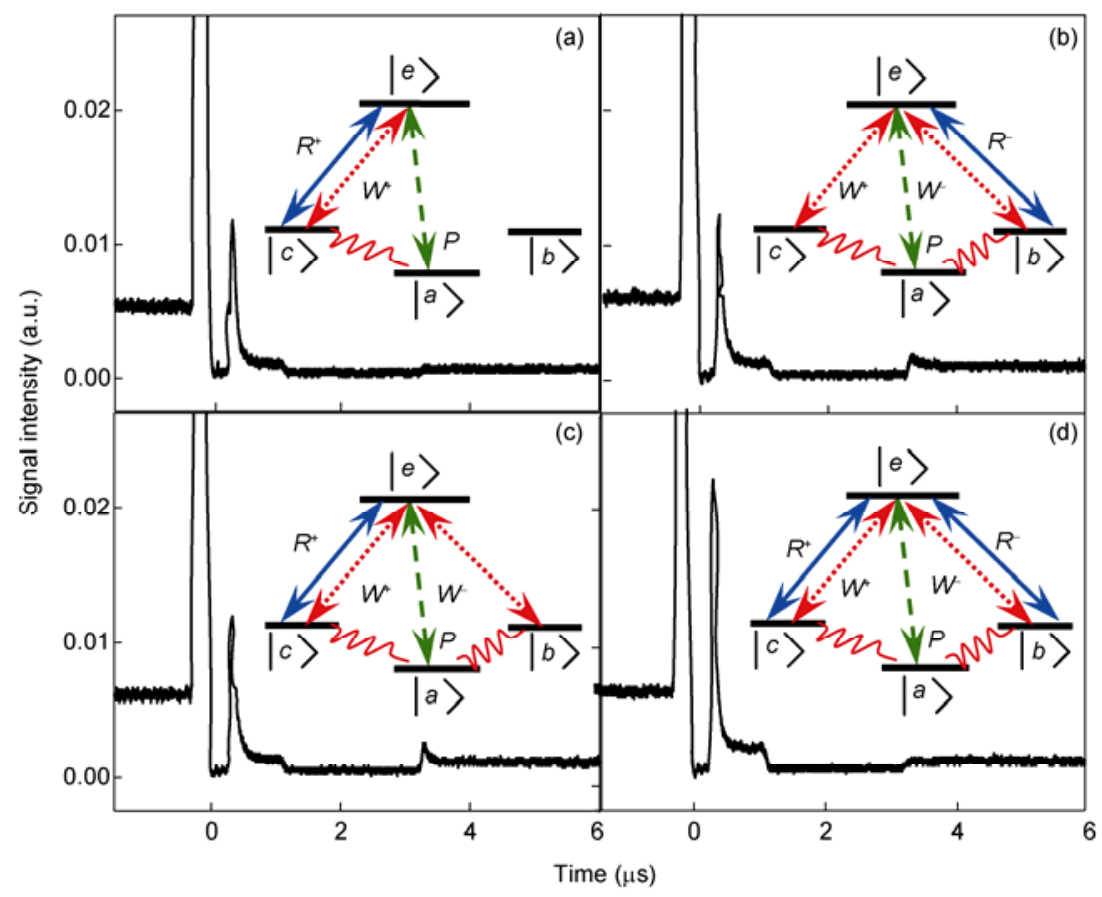

Figure 7 (Color online) Measured results for different storage-release cases. (a) The writing beam is right circularly polarized, and the reading beam is also right circularly polarized. (b)-(d) The writing beam is linearly polarized (consisting of left and right circularly polarized), and in (b) the reading beam is left circularly polarized, in (c) the reading beam is right circularly polarized, and in (d) the right and left circularly polarized reading beams are both present, and the total phase difference $\delta \phi$ is approximately 0 . 
$\tilde{\sigma}_{b a}$ ). If a single reading beam (either $R^{+}$or $R^{-}$) is used, the readout signal is approximately the same as in the single-channel case. Figure 7(b) and (c) present the readout signals from the individual memory channels $\tilde{\sigma}_{b a}$ and $\tilde{\sigma}_{c a}$ (with only one reading beam on), respectively. When both reading beams $\left(R^{+}\right.$and $\left.R^{-}\right)$are on simultaneously and the total phase is tuned to satisfy $\Delta=0$ by adjusting the relative phase between the two reading beams $\left(\delta_{R}\right)$, a maximum readout signal is obtained (Figure 7(d)). The retrieved signal intensity is twice that of the single channel case for the same storage time of $380 \mathrm{~ns}$, as indicated in the calculations. These results clearly show that the quantum interference between the two stored collective SWEs was mediated by the coherent reading light beams.

When the total phase difference $\Delta$ is adjusted to be $\pi$ by tuning $\delta_{R}$, no optical signal is retrieved from the atomic medium, even though both of the reading beams are turned on at $380 \mathrm{~ns}$. After a delay time $\tau$ of $3.4 \mu \mathrm{s}$, the atomic medium is read again with other (writing) laser beams, as shown in Figure 8(a). For comparison, Figure 8(b) presents the readout results when the stored SWEs were not perturbed before the system was read at $3.4 \mu$ s storage time. These results indicate that although the stored SWEs have been read with two reading beams at $380 \mathrm{~ns}$ for $\Delta=\pi$, they have not been converted into optical signals because of destructive quantum interference between them and they have remained in the atomic medium, until they are read again with other laser beams at a later time.

Figure 9 (square points) shows the total retrieved signal as a function of $\delta_{R}$ at $\tau=380 \mathrm{~ns}$. For a fixed $\delta_{w}=\pi / 2$ and Larmor precession (e.g. $2 \Omega_{L} \tau=$ constant), $\delta_{R}$ is proportional to $\Delta$. The retrieval efficiency shows a sinusoidal interference pattern as $\delta_{R}$ is varied (see eq. (8)). At the same time, this relationship can be viewed as the population

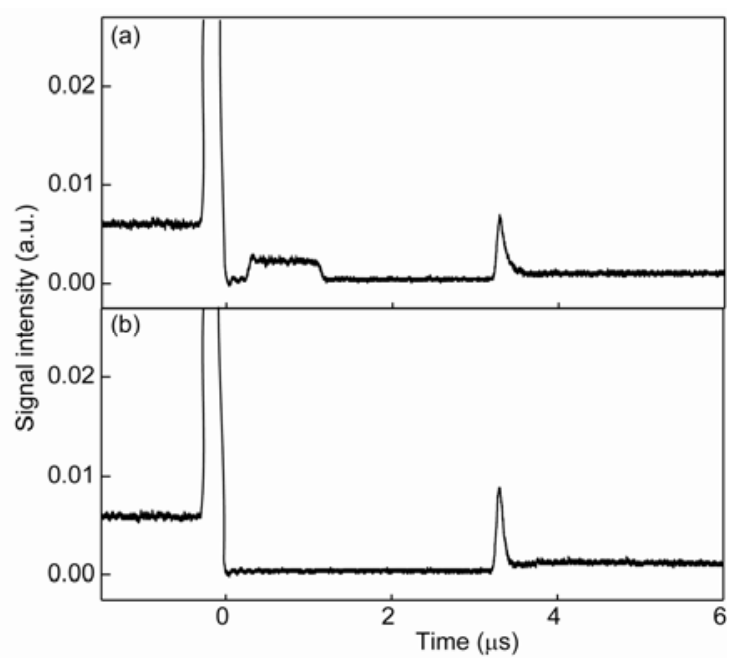

Figure 8 Measured retrieved results for the second released signals. projected into the given state, $\hat{\sigma}_{+}^{\delta_{R}}|a\rangle$, and the interference pattern shows that the population in the $\hat{\sigma}_{+}^{\delta_{R}}|a\rangle$ state depends on the relative phase $\delta_{R}$ between the two basis states. After the stored collective SWEs are first read by a pair of reading beams (at $380 \mathrm{~ns}$ ), the atomic medium is read again by a horizontally-polarized (writing) laser beam at $3.4 \mu \mathrm{s}$. The signals retrieved at $3.4 \mu \mathrm{s}$ are also shown in Figure 9 (circular points) as a function of $\delta_{R}$ (for the first pair of reading beams). It is clear that if all of the stored SWEs in $\hat{S}(z, t)$ are mapped completely into the signal field at the earlier time (380 ns), the second reading by the second beam will not be able to retrieve any information. However, if the first reading at $t=380 \mathrm{~ns}$ did not retrieve information from the SWEs (because $\Delta=0$ ), the second reading at $3.4 \mu$ s will have the maximum readout signal.

In the tripod atomic system with a small magnetic field $(\sim 300 \mathrm{mG})$, the Zeeman sublevels $|b\rangle$ and $|c\rangle$ are split, which causes the Larmor precession for the stored collective SWEs. The total readout signal intensity as a function of storage time shows sinusoidal behavior, as shown in Figure 10 (circular points). However, such oscillatory behavior in the readout intensity can severely limit the ability to retrieve arbitrarily polarized states, and the ability to probe longlived entanglements between photons and SWEs at any desired time. Using the current phase-sensitive storage/ retrieval scheme, we demonstrate that by adjusting the relative phase difference between the two reading beams, this "collapse and revival" behavior in the readout signal intensity caused by the Larmor precession can be completely compensated (i.e. $\delta_{R}$ can compensate $2 \Omega_{L} \tau$ in a total phase difference $\Delta$ ), as shown in Figure 10 (square points). In this case, the maximum readout signal can be accessed at any storage time, and controlled by adjusting the relative

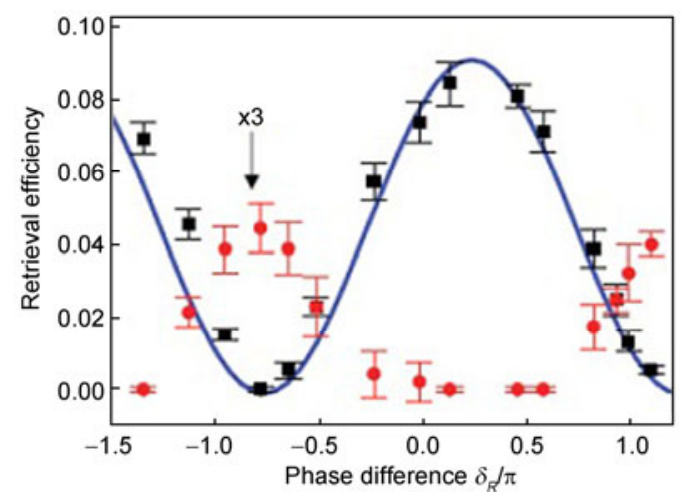

Figure 9 (Color online) Retrieval efficiency as a function of the relative phase $\delta_{R}$. The blue square points are the total retrieved signal intensities at $380 \mathrm{~ns}$ storage time for different values of $\Delta$. The solid blue line is the best fit to the data. The red circular points are the retrieved signal intensities at $3.4 \mu$ s storage time, after the system was first read at $380 \mathrm{~ns}$ with the pair of reading beams. 


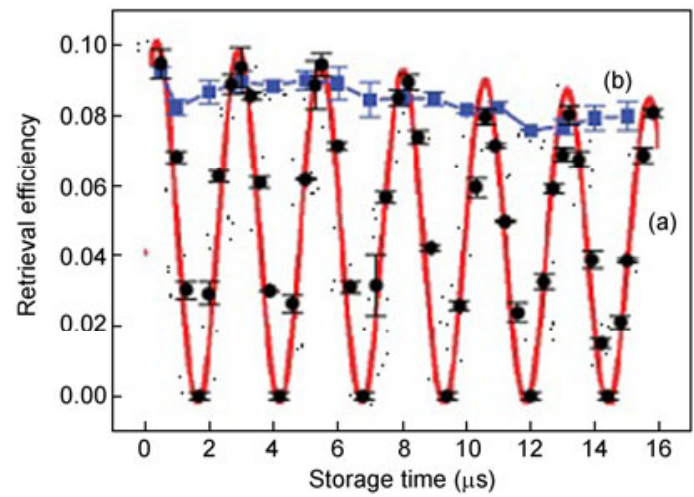

Figure 10 (Color online) Retrieval efficiency and compensation of Larmor precession. Black circular points: retrieval efficiency as a function of storage time. Curve (a) (red curve): fitting to the function $R_{e}=$

$A \cos ^{2}\left(\Omega_{L} t-\varphi / 2\right) \mathrm{e}^{-t / t_{0}}$, where the fitting parameters are $A=0.1, \Omega_{L}=$ $2 \pi \times 0.21 \mathrm{MHz}, \varphi=0.3 \pi$ (initial phase), and the lifetime $t_{0} \approx 90 \mu \mathrm{s}$. Curve (b) (with blue squares): the results with compensated Larmor precession by adjusting the relative phase $\delta_{R}$.

phase difference between the two reading beams.

The above two-channel storage scheme and coherent phase-sensitive readout technique have significant advantages in extending single-channel memory systems for quantum information processing, especially for qubit memory, and have overcome some important obstacles in using atomic ensembles as optical memory devices. This two-channel storage/retrieval scheme provides a unique way to measure the relative phase of the qubit memory and probe the population in a given coherent superposition state. The accessed Rabi oscillation (with a lifetime of $90 \mu \mathrm{s}$ ) between two superposition states of the SWEs has potential for application to performing qubit gate operations. Also, by compensating the Larmor precession by tuning the relative phase between the two reading beams, the collapse and revival behavior of the total readout signal is eliminated and the total readout signal is maintained at its maximum for more than $100 \mu \mathrm{s}$. This phase compensation method can also be applied to experiments for the quantum memory for photon polarization states and entanglement of photons and atomic ensembles. In these experiments, if we could first measure the evolution of the relative phase of the qubit memory caused by the Larmor precession, and alter the relative phase between the two retrieved orthogonally-polarized photons using an EOM according to the measured phase data, then the changes in the photon polarization states because of the Larmor precession could be compensated, and therefore the memory time would be significantly increased.

\section{Coherent manipulation of spin wave vector for polarization of photons in an atomic ensemble}

The coherent manipulation of the quantum states in memory elements plays an important role in QIP [2,32,33]. Atomic ensembles with the ability for collectively enhanced coupling to a particular light mode [2] can serve as good quantum memory elements and have attracted considerable attention in recent years. Recent experimental studies of the collective qubit memory used to achieve atom-photon entanglement have made great progress. In these studies [34-36], two orthogonal [34] or two spatially distinct [35] spin waves, or two atomic ensembles sharing a spin-wave excitation [36], are used to encode the long-time atomic qubit. The lifetime of the qubit memory for an optical lattice spin wave has reached $3 \mathrm{~ms}$ [34]. QIP schemes based on atomic ensembles such as entanglement swapping [32], the multipartite entanglement of atomic ensembles [33,37], and the controlled-NOT gate [37] have been proposed. In these proposed schemes, the memory qubits may be encoded in two orthogonal spin waves, and single-bit operations are required. These single qubit gate operations are the $R(\theta, \varphi)$ and $R_{z}\left(\phi_{z}\right)$ rotations [38], which can be used to build an arbitrary rotation on the Bloch sphere. The single qubit operations have been realized in a single ion [38], in trapped neutral atoms [39,40], or in a quantum dot system [41].

In 2011, we demonstrated an experiment on the two unitary rotations $R(\theta, \varphi)$ and $R_{z}\left(\phi_{z}\right)$ on the basis of their formation by two orthogonal spin waves in an atomic ensemble [12]. The relevant ${ }^{87} \mathrm{Rb}$ levels involved in the storage, Raman manipulation and readout processes are shown in Figure 11(a)-(c), respectively, where $|\uparrow\rangle=$ $\left|5^{2} S_{1 / 2}, F=1, M_{F}=+1\right\rangle, \quad|\downarrow\rangle=\left|5^{2} S_{1 / 2}, F=1, M_{F}=-1\right\rangle$, $|s\rangle=\left|5^{2} S_{1 / 2}, F=2, M_{F}=+1\right\rangle \quad$ a n d $\quad|e\rangle=\mid 5^{2} P_{1 / 2}, F^{\prime}=1$, $\left.M_{F}=0\right\rangle$. The spin wave associated with the coherence between the state $|s\rangle$ and the superposition state $|\Phi\rangle=\alpha|\uparrow\rangle+\mathrm{e}^{-i \Delta} \beta|\downarrow\rangle$ (where $|\alpha|^{2}+|\beta|^{2}=1$ ) is written as the vector $\hat{S}_{s \Phi}(z, t)=\left\langle\left|\tilde{\rho}_{s \Phi}(z, t)\right|\right\rangle\left(\begin{array}{c}\alpha \\ \mathrm{e}^{-i \Delta} \beta\end{array}\right)$, where $\hat{S}_{s \uparrow}(z, t)=$ $\left\langle\left|\tilde{\rho}_{s \Phi}(z, t)\right|\right\rangle\left(\begin{array}{c}\alpha \\ 0\end{array}\right)\left(\hat{S}_{s \downarrow}(z, t)=\left\langle\left|\tilde{\rho}_{s \Phi}(z, t)\right|\right\rangle\left(\begin{array}{l}0 \\ \beta\end{array}\right)\right)$ is the spin wave component associated with the coherence between the state $|s\rangle$ and the state $|\uparrow\rangle(|\downarrow\rangle)$. For an initial condition of $\Phi(0)=|\uparrow\rangle$, the spin wave is expressed as $\hat{S}(z, 0)=\left\langle\left|\tilde{\rho}_{s \uparrow}(z, 0)\right|\right\rangle\left(\begin{array}{l}1 \\ 0\end{array}\right)$. The coherent population can be transferred between the $\hat{S}_{s \uparrow}(z, t)$ and $\hat{S}_{s \downarrow}(z, t)$ channels via a two-photon Raman transition. The Raman laser beam consists of $\sigma^{+}$- and $\sigma^{-}$-polarized light fields $E_{R_{+}}$and $E_{R_{-}}$, which couple to the $|\uparrow\rangle \leftrightarrow|e\rangle$ and $|\downarrow\rangle \leftrightarrow|e\rangle$ transitions. The unitary Raman rotation $R(\theta, \phi)$ can be written as 


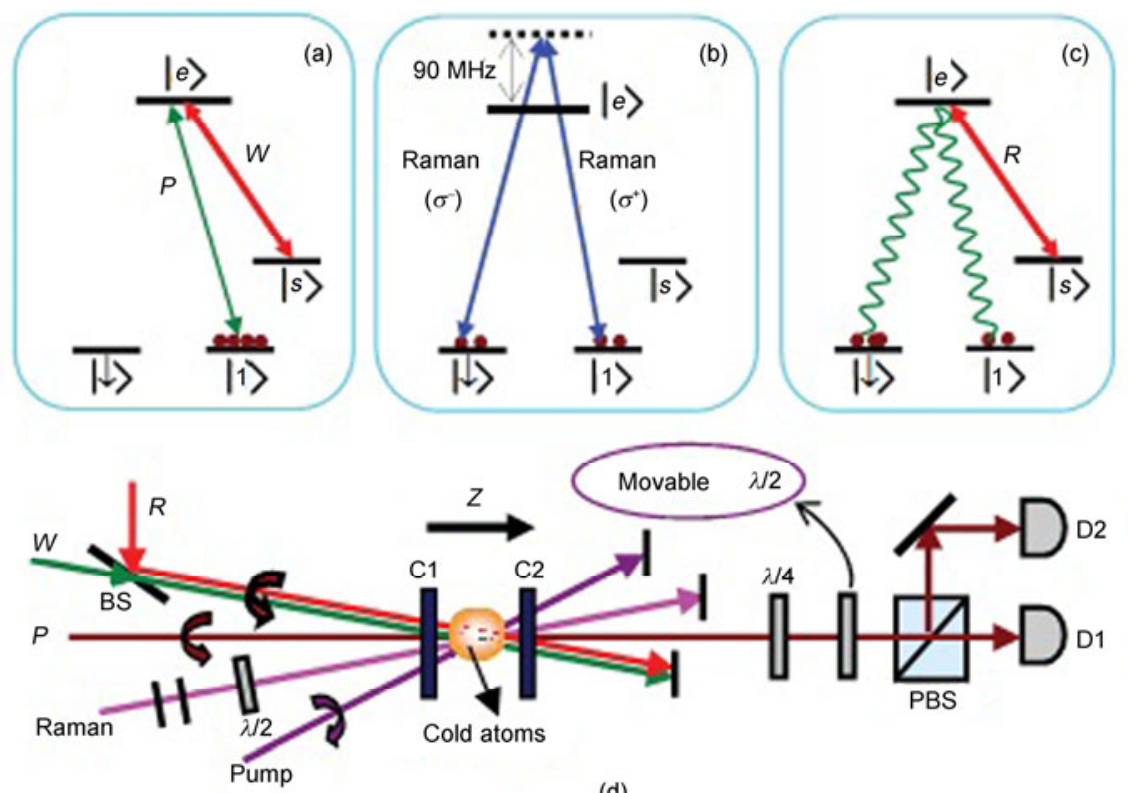

(d)

Figure 11 (Color online) The experimental scheme for rotation of the spin-wave vector. (a)-(c) Atomic level structures of the ${ }^{87} \mathrm{Rb}$ atom for optical storage, Raman manipulation, and retrieval. (d) Experimental setup, where $R, W$, and $P$ denote reading, writing, and probe laser beams, respectively; BS, beam splitter; $\mathrm{C} 1$ and $\mathrm{C} 2$, coils; PBS, polarization beam splitter; D1 and D2, photodetectors.

$R(\theta, \phi)=\left(\begin{array}{cc}\cos \frac{\theta}{2} & -i \mathrm{e}^{i \phi} \sin \frac{\theta}{2} \\ -i \mathrm{e}^{-i \phi} \sin \frac{\theta}{2} & \cos \frac{\theta}{2}\end{array}\right)$, where $\theta=\Omega_{R} t$ and

$\phi=\phi_{+}-\phi_{-}$is the phase difference between the Raman fields $E_{R+}$ and $E_{R_{-}}$.

In a magnetic field, the two spin-wave components $\hat{S}_{s \uparrow}(z, t)$ and $\hat{S}_{s \downarrow}(z, t)$ experience the Larmor precession and get the phase shifts $\psi_{1}$ and $\psi_{2}$ respectively, which introduce the relative phase $\varphi_{z}=\psi_{1}-\psi_{2}$ after an evolution time interval $t$. The evolution of the spin wave is described by $\hat{S}(z, t)=R_{z}\left(\varphi_{z}\right) \hat{S}(z, 0)=\left\langle\left|\tilde{\rho}_{s \uparrow}(z, 0)\right|\right\rangle\left(\begin{array}{l}\alpha \\ \mathrm{e}^{i \varphi_{z}} \beta\end{array}\right)$, where $R_{z}\left(\varphi_{z}\right)=$ $\left(\begin{array}{cc}1 & 0 \\ 0 & \mathrm{e}^{i \varphi_{2}}\end{array}\right)$ is the matrix rotation, and the initial spin wave is assumed to be $\hat{S}(z, 0)=\left\langle\left|\tilde{\rho}_{s \uparrow}(z, 0)\right|\right\rangle\left(\begin{array}{l}\alpha \\ \beta\end{array}\right)$. It is obvious that the spin wave acquires a relative phase of $\Delta=\varphi_{z}$ because of the Larmor precession.

We used a cold ${ }^{87} \mathrm{Rb}$ atomic cloud to serve as the atomic ensemble. The measured optical depth of the cold atoms is about 1.5 and the trap temperature can reach $\sim 200 \mu \mathrm{K}$ (see [12] for the experimental details).

To perform the spin wave $R(\theta, \varphi)$ rotation induced by a Raman laser pulse, we first observe the population transfer efficiency from $\hat{S}_{s \uparrow}(z, t)$ to $\hat{S}_{s \downarrow}(z, t)$ components as a function of the Rabi frequency of the Raman laser beam. Figure 12 (the square (circle) points) presents the normalized retrieval efficiency $N_{+}\left(N_{-}\right)$as a function of the Raman-Rabi frequency $\Omega_{R}$, and the solid curves in Figure 12 are the best fits of the functions $\left(1 \pm \cos 2 \pi B \Omega_{R} \tau_{R}\right) / 2$, with the parameter $B=0.82$ from the experimental data. These curve fits present sinusoidal Rabi oscillations which are consistent with the theoretical predictions of $N_{ \pm}=$ $\left(1 \pm \cos 2 \pi \Omega_{R} \tau_{R}\right) / 2$. At $\theta=2 \pi B \Omega_{R} \tau_{R}=\pi$ (the upper axis in Figure 12), the population transfer efficiency reaches $\sim 97 \%$.

By applying a variable field of the magnetic pulse $B(t)$ to the atomic ensemble, we implement a spin-wave phase

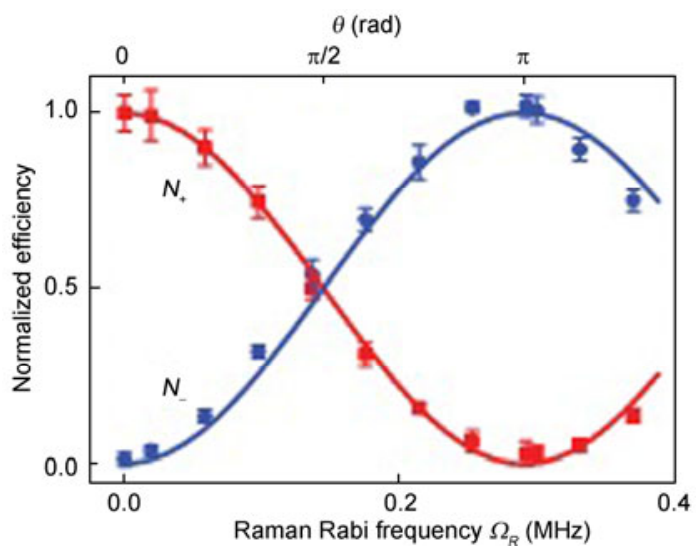

Figure 12 (Color online) The coherent population transfer efficiency between the two spin-wave components as a function of the Rabi frequency of the Raman laser beam. 
operation, i.e. $R_{z}\left(\varphi_{z}\right)=\left(\begin{array}{cc}1 & 0 \\ 0 & \mathrm{e}^{i \varphi_{z}}\end{array}\right)$ rotation. We apply a current pulse $I(t)$ to a pair of coils, $\mathrm{C} 1$ and $\mathrm{C} 2$, to generate the pulsed magnetic field $B(t)$. The generated pulsed magnetic field $B(t)$ is proportional to the current $I(t)$. By changing the area $\int I(t) \mathrm{d} t$ of the current pulse, we can control the changes in $\Delta \varphi_{z}$ and then implement the $R_{z}\left(\Delta \varphi_{z}\right)$ rotation. By observing the interference fringe between the readout fields $\varepsilon_{+}^{\text {out }}(t)$ and $\varepsilon_{-}^{\text {out }}(t)$, we verify the implementation of the $R_{z}\left(\Delta \phi_{z}\right)$ rotation. In the experiment, we first apply a $\pi / 2$ linearly-polarized Raman laser pulse (with an orientation angle of $\varphi_{R}=8^{\circ}$ ) and then apply a magnetic pulse with a duration of $\tau \sim 7 \mu$ s to rotate the initial spin wave $\tilde{S}(z, 0)$. After these operations, the spin wave evolves into $\tilde{S}(z, t)=\frac{\left\langle\left|\tilde{\rho}_{s \uparrow}(z, 0)\right|\right\rangle}{\sqrt{2}}\left(\begin{array}{c}1 \\ \mathrm{e}^{i \Delta_{0}+i \Delta \varphi_{z}}\end{array}\right), \quad$ where $\quad \Delta_{0}=-\pi / 2-\phi$ $+\varphi_{z 0}$ is kept constant. At $t=17 \mu \mathrm{s}$, we turned on the reading beam to read the spin wave. Figure 13 plots the relative retrieved efficiencies $N_{ \pm 45^{\circ}}$ (square and circular dots) as a function of the relative magnetic pulse area $A$ [12]. The solid lines are the curve fits of the sinusoidal functions $(a \pm b \cos 2 \pi A) / 2$ to the measured data $N_{ \pm 45^{\circ}}$, with parameters $\Delta_{0}=0, a=0.93$ and contrast $b=0.93$. The magnetic pulse width is $\sim 7 \mu$ s in the experiment presented here, and we believe that it can be further decreased by improving the coils and the current pulse source.

To estimate the $\pi / 2$-pulse fidelity, we performed a Ramsey experiment by applying two linearly-polarized $\pi / 2 \mathrm{Ra}$ man laser pulses with a variable time interval $\tau_{R}$. At $t=17 \mu \mathrm{s}$, we retrieve the spin wave $\hat{S}(z, t)$ into the readout signal fields $\varepsilon_{ \pm}^{\text {out }}$. Figure 14 plots the measured relative photon numbers $N_{ \pm}$as a function of the interval $\tau_{R}$. The solid lines are the curve fits of the sinusoid function $\left(1 \pm b_{ \pm} \cos 2 \pi \tau_{R} / T_{L}\right) / 2$ to the data $N_{ \pm}$, with a Larmor period of $T_{L}=2.82 \times 10^{-6} \mathrm{~s}$, and contrasts $b_{+}=1$ for $N_{+}$, as well as $b_{-}=0.85$ for $N_{-}$. According to [13], we calculate the $\pi / 2$

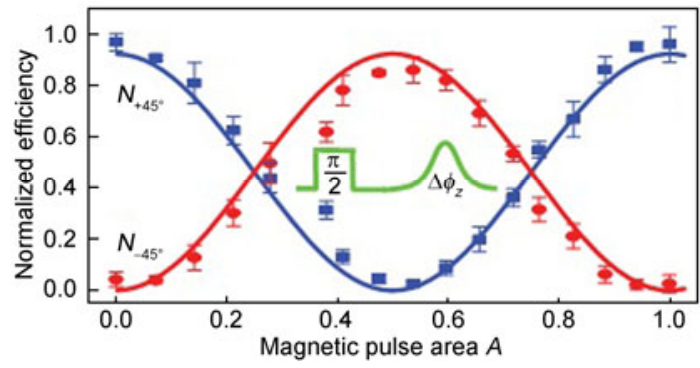

Figure 13 (Color online) The normalized retrieval efficiencies as a function of the magnetic pulse area $A$.

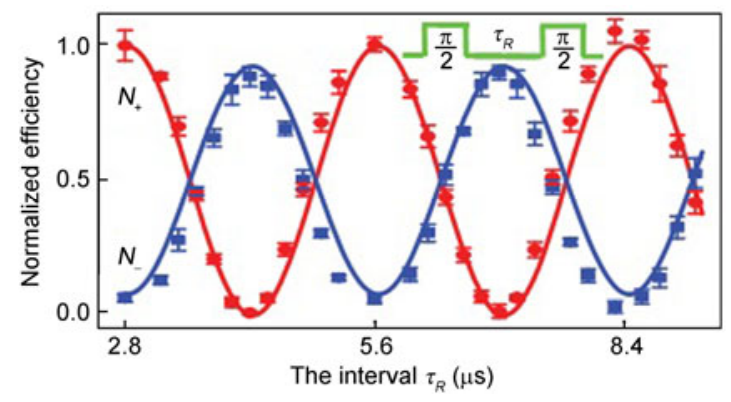

Figure 14 (Color online) Ramsey fringes for a pair of $\pi / 2$ pulses with a variable separation time $\tau_{R}$. Red circular points (blue square points): the normalized retrieval efficiency $N_{+}\left(N_{-}\right)$. The solid lines are sinusoidal fits to the data.

fidelity using the lower value of contrast $\left(b_{-}\right)$, which is $F_{\pi / 2}=(1+\sqrt{b}) / 2 \approx 96 \%$.

The above experiments present the $R(\theta, \varphi)$ and $R_{z}\left(\Delta \phi_{z}\right)$ rotations of the spin-wave vector on the Bloch sphere when Raman laser and magnetic field pulses are applied. For the case where the initially stored spin wave has an atomic collective excitation (i.e. $N \int\left\langle\tilde{\rho}_{s \uparrow}(z, 0) \tilde{\rho}_{s \uparrow}^{+}(z, 0)\right\rangle \mathrm{d} z=1$ ), the two spin-wave components sharing a single excitation become the two basis states of a qubit. The manipulation demonstrated in the work presented here may thus be used to implement a single qubit gate operation, which has potential applications in QIP based on an atomic ensemble.

In conclusion, we have briefly summarized the experimental studies conducted recently by our group on enhanced cross-phase modulation based on double EIT, the quantum interference of stored dual-channel spin-wave excitations and the coherent manipulation of the spin wave vector for polarization of photons in a single tripod atomic system. The focus of this research is on the development of a long lifetime quantum memory for optical information in atomic ensembles.

This work was supported by the National Basic Research Program of China (2010CB923103) and the National Natural Science Foundation of China (10874106, 60821004 and 10904086).

1 Lukin M D, Imamoglu A. Controlling photons using electromagnetically induced transparency. Nature, 2001, 413: 273-276

2 Lukin M D. Trapping and manipulating photon states in atomic ensembles. Rev Mod Phys, 2003, 75: 457-472

3 Liu C, Dutton Z, Behroozi C H, et al. Observation of coherent optical information storage in an atomic medium using halted light pulses. Nature, 2001, 409: 490-493

4 Phillips D F, Fleischhauer A, Mair A, et al. Storage of light in atomic vapor. Phys Rev Lett, 2001, 86: 783-786

5 Van der Wal C H, Eisaman M D, André A, et al. Atomic memory for correlated photon states. Science, 2003, 301: 196-200

6 Matsukevich D N, Chanelière T, Bhattacharya M, et al. Entanglement of a photon and a collective atomic excitation. Phys Rev Lett, 2005, 95: 040405

7 De Riedmatten H, Laurat J, Chou C W, et al. Direct measurement of 
decoherence for entanglement between a photon and stored atomic excitation. Phys Rev Lett, 2006, 97: 113603

8 Zhao R, Dudin Y O, Jenkins S D, et al. Long-lived quantum memory. Nat Phys, 2009, 5: 100-104

9 Zhao B, Chen Y A, Bao X H, et al. A millisecond quantum memory for scalable quantum networks. Nat Phys, 2009, 5: 95-99

10 Li S J, Yang X D, Cao X M, et al. Enhanced cross-phase modulation based on a double electromagnetically induced transparency in a four-level tripod atomic system. Phys Rev Lett, 2008, 101: 073602

11 Wang H, Li S J, Xu Z X, et al. Quantum interference of stored dual-channel spin-wave excitations in a single tripod system. Phys Rev A, 2011, 83: 043815

$12 \mathrm{Li} \mathrm{S} \mathrm{J,} \mathrm{Xu} \mathrm{Z} \mathrm{X,} \mathrm{Zheng} \mathrm{H} \mathrm{Y,} \mathrm{et} \mathrm{al.} \mathrm{Coherent} \mathrm{manipulation} \mathrm{of}$ spin-wave vector for polarization of photons in an atomic ensemble. Phys Rev A, 2011, 84: 043430

13 Turchette Q A, Hood C J, Lange W, et al. Measurement of conditional phase shifts for quantum logic. Phys Rev Lett, 1995, 75: 4710-4713

14 Rebić S, Vitali D, Ottaviani C, et al. Polarization phase gate with a tripod atomic system. Phys Rev A, 2004, 70: 032317

15 Joshi A, Xiao M. Phase gate with a four-level inverted-Y system. Phys Rev A, 2005, 72: 062319

16 Lukin M D, Imamoğlu A. Nonlinear optics and quantum entanglement of ultraslow single photons. Phys Rev Lett, 2000, 84: 1419-1422

17 Nemoto K, Munro W J. Nearly deterministic linear optical controlled-NOT gate. Phys Rev Lett, 2004, 93: 250502

18 Barrett S D, Kok P, Nemoto K, et al. Symmetry analyzer for nondestructive Bell-state detection using weak nonlinearities. Phys Rev A, 2005, 71: 060302(R)

19 Munro W J, Nemoto K, Spiller T P. Weak nonlinearities: A new route to optical quantum computation. New J Phys, 2005, 7: 137

20 Schmidt H, Imamogdlu A. Giant Kerr nonlinearities obtained electromagnetically induced transparency. Opt Lett, 1996, 21: 1936-1938

21 Kang H, Zhu Y F. Observation of large Kerr nonlinearity at low light intensities. Phys Rev Lett, 2003, 91: 093601

22 Harris S E, Hau L V. Nonlinear optics at low light levels. Phys Rev Lett, 1999, 82: 4611-4614

23 Petrosyan D, Kurizki G. Symmetric photon-photon coupling by atoms with Zeeman-split sublevels. Phys Rev A, 2002, 65: 033833

24 Wang Z B, Marzlin K P, Sanders B C. Large cross-phase modulation between slow copropagating weak pulses in ${ }^{87} \mathrm{Rb}$. Phys Rev Lett, 2006, 97: 063901

25 Li S J, Yang X D, Cao X M, et al. Two electromagnetically induced transparency windows and an enhanced electromagnetically induced transparency signal in a four-level tripod atomic system. J Phys B: At
Mol Opt Phys, 2007, 40: 3211-3219

26 Schnorrberger U, Thompson J D, Trotzky S, et al. Electromagnetically induced transparency and light storage in an atomic Mott insulator. Phys Rev Lett, 2009, 103: 033003

27 Zhang R, Garner S R, Hau L V. Creation of long-term coherent optical memory via controlled nonlinear interactions in Bose-Einstein condensates. Phys Rev Lett, 2009, 103: 233602

28 Matsukevich D N, Chanelière T, Bhattacharya M, et al. Entanglement of a photon and a collective atomic excitation. Phys Rev Lett, 2005, 95: 040405

29 Joshi A, Xiao M. Generalized dark-state polaritons for photon memory in multilevel atomic media. Phys Rev A, 2005, 71: 041801(R)

30 Raczyński A, Zaremba J, Zielińska-Kaniasty S. Beam splitting and Hong-Ou-Mandel interference for stored light. Phys Rev A, 2007, 75: 013810

31 Wang B, Han Y X, Xiao J T, et al. Preparation and determination of spin-polarized states in multi-Zeeman-sublevel atoms. Phys Rev A, 2007, 75: 051801(R)

32 Duan L M, Lukin M D, Cirac J I, et al. Long-distance quantum communication with atomic ensembles and linear optics. Nature, 2001, 414: 413-418

33 Duan L M. Entangling many atomic ensembles through laser manipulation. Phys Rev Lett, 2002, 88: 170402

34 Dudin Y O, Jenkins S D, Zhao R, et al. Entanglement of a photon and an optical lattice spin wave. Phys Rev Lett, 2009, 103: 020505

35 Chen S, Chen Y A, Zhao B, et al. Demonstration of a stable atom-photon entanglement source for quantum repeaters. Phys Rev Lett, 2007, 99: 180505

36 Tanji H, Ghosh S, Simon J, et al. Heralded single-magnon quantum memory for photon polarization states. Phys Rev Lett, 2009, 103: 043601

37 Dong P, Xue Z Y, Yang M, et al. Generation of cluster states. Phys Rev A, 2006, 73: 033818

38 Hanneke D, Home J P, Jost J D, et al. Realization of a programmable two-qubit quantum processor. Nat Phys, 2010, 6: 13-16

39 Yavuz D D, Kulatunga P B, Urban E, et al. Fast ground state manipulation of neutral atoms in microscopic optical traps. Phys Rev Lett, 2006, 96: 063001

40 Jones M P A, Beugnon J, Gaëtan A, et al. Fast quantum state control of a single trapped neutral atom. Phys Rev A, 2007, 75: 040301

41 Press D, Ladd T D, Zhang B Y, et al. Complete quantum control of a single quantum dot spin using ultrafast optical pulses. Nature, 2008, 456: $218-221$

Open Access This article is distributed under the terms of the Creative Commons Attribution License which permits any use, distribution, and reproduction in any medium, provided the original author(s) and source are credited. 